\title{
Autophagy: A potential target for thyroid cancer therapy (Review)
}

\author{
HEQING YI ${ }^{1}$, BIN LONG ${ }^{1}$, XUEMEI YE ${ }^{1}$, LIJUN ZHANG ${ }^{1}$, \\ XIAODONG LIU $^{2}$ and CHUNYAN ZHANG ${ }^{1}$ \\ ${ }^{1}$ Department of Nuclear Medicine, Zhejiang Cancer Hospital, Hangzhou, Zhejiang 310022; \\ ${ }^{2}$ Key Laboratory of Radiobiology (Ministry of Health), School of Public Health, \\ Jilin University, Changchun, Jilin 130021, P.R. China
}

Received November 26, 2013; Accepted May 29, 2014

DOI: $10.3892 / \mathrm{mco} .2014 .305$

\begin{abstract}
The sharply increasing incidence of thyroid cancer has attracted considerable attention over the last few years. The combination of surgery, radioiodine ablation and thyroid-stimulating hormone suppression is usually efficient for the majority of thyroid tumors. However, advanced thyroid cancer that is recurrent, metastatic and ${ }^{131}$ I-refractory, or medullary thyroid cancer, pose a therapeutic challenge. Autophagy is a process that metabolizes damaged cytoplasmic organelles and long-lived proteins in order to recycle cellular materials and maintain homeostasis. It has been confirmed that autophagy plays a dual role during cancer development, progression and treatment, mainly depending on the type and stage of the tumor. Autophagy modulation has become a potential therapeutic target for diverse diseases. The mechanism of thyroid tumorigenesis and cancer progression was largely demonstrated to be correlated with the dysregulation of the Ras/Raf/mitogenactivated protein kinase kinase/extracellular signal-regulated kinase and the phosphoinositide 3-kinase/Akt/mammalian target of rapamycin pathways, as well as with abnormal epigenetic modifications. Those mechanisms are associated with autophagy regulation and may be beneficial for the treatment of advanced thyroid cancer. However, the number of available studies on the role of autophagy in thyroid cancer development, progression and treatment outcome, is currently limited. The aim of this review was to elaborate on the relevant knowledge and future prospectives of autophagy in the treatment of thyroid cancer.
\end{abstract}

Correspondence to: Dr Chunyan Zhang, Department of Nuclear Medicine, Zhejiang Cancer Hospital, 38 Guangji Road, Hangzhou, Zhejiang 310022, P.R. China

E-mail: zhangchunyan_hz@126.com

Dr Xiaodong Liu, Key Laboratory of Radiobiology (Ministry of Health), School of Public Health, Jilin University, 1163 Xinmin Street, Changchun, Jilin 130021, P.R. China

E-mail: liuxd2002@126.com

Key words: thyroid cancer, autophagy, epigenetics, phosphoinositide 3-kinase/Akt

\section{Contents}

1. Introduction

2. Development and progression of thyroid cancer

3. Treatment strategies for thyroid cancer

4. Role of autophagy in cancer

5. Conclusion

\section{Introduction}

According to data published by the USA National Cancer Institute (http://seer.cancer.gov/statfacts/html/thyro.html), the incidence of thyroid cancer has increased over the last few years, from 7.3 per 100,000 individuals in 1999 , to 13.3 per 100,000 individuals in 2010. In total, 44,670 new cases of thyroid cancer were diagnosed and 1,690 patients succumbed to thyroid cancer in the USA in 2010 (1). The estimated data indicated that there were 60,220 new cases expected in 2013, accounting for $3.6 \%$ of all new cancer cases. There were 1,850 estimated thyroid cancer-related deaths in 2013, accounting for $0.3 \%$ of all cancer deaths. There are currently $\sim 534,973$ thyroid cancer patients in the USA. The incidence of thyroid cancer is on the increase and thyroid cancer is currently a common, life-threatening malignancy and a major health concern worldwide.

Thyroid cancer includes differentiated thyroid cancer (DTC), poorly differentiated and undifferentiated thyroid cancer. Papillary thyroid cancer (PTC), accounting for $80-85 \%$ and follicular thyroid cancer (FTC), accounting for $10-25 \%$ of the cases, are the most common subtypes of DTC, with Hürthle cell thyroid cancer, originating from thyroid follicular cells, accounting for 1-2\% of DTCs (2). Anaplastic thyroid cancer (ATC), accounting for $\sim 1.7 \%$ of the cases, is a subtype of thyroid cancer that develops from DTC, which has lost its iodine uptake ability, has become highly aggressive and is associated with a poor prognosis, with a median survival of $\sim 5$ months and an 1-year survival rate of $20 \%(3,4)$. Another type of thyroid cancer is medullary thyroid cancer (MTC), accounting for $2-8 \%$ of the cases. MTC originates from parafollicular cells, which produce the hormone calcitonin $(5,6)$, and its overall 10-year survival rate is $90 \%$ for local tumors, whereas for metastatic MTCs this percentage decreases to $40 \%$ (7). Another study on metastatic 
MTC reported a median survival of 1 year (5). DTC cells retain the characteristic of iodine uptake, similar to normal thyroid gland cells. Radioiodine $\left({ }^{131} \mathrm{I}\right)$ emits $\beta$-rays and has a similar biological function with common iodine. ${ }^{131} \mathrm{I}$ is used for the treatment of the recurrent and metastatic disease and for the ablation of residual DTC tissue. The most effective treatment approach for DTC is considered to be surgery + ${ }^{131} \mathrm{I}+$ thyroid-stimulating hormone (TSH) suppression, which was found to be effective for the majority of DTCs. However, $\sim 5 \%$ of DTCs lose their ability to uptake ${ }^{131} \mathrm{I}$ and progress to ${ }^{131} \mathrm{I}$-refractory tumors (8). The majority of ${ }^{131} \mathrm{I}$-refractory DTCs have a poor prognosis, with a survival time of $\leq 3$ years (9). For such patients, the therapeutic options remain limited.

\section{Development and progression of thyroid cancer}

A review of the molecular studies on thyroid cancer demonstrated that the Ras/Raf/mitogen-activated protein kinase kinase/extracellular signal-regulated kinase (also referred to as mitogen-activated protein kinase; MAPK) and the phosphoinositide 3-kinase (PI3K)/Akt signaling pathways are key to the development and progression of thyroid cancer, which are associated with tumorigenesis, proliferation, apoptosis, angiogenesis, metastasis and metabolism $(2,5,10,11)$. The MAPK signaling pathway involves a series of serine/threonine protein kinases that convert extracellular stimuli into a wide range of cellular responses through transmembrane protein receptors, such as epidermal growth factor receptor/vascular endothelial growth factor (VEGF)/rearranged during transfection (RET) $(12,13)$. The activation of a tyrosine kinase leads to a cascade of sequential phosphorylations in the Ras/Raf/MEK/ERK pathway that promote thyroid tumorigenesis, as it has been observed in patients with DTC $(10,13)$. The expression of VEGF, which was found to be increased in thyroid cancer tissue compared to normal thyroid tissue, promotes vessel formation in thyroid cancer and shortens the progression-free survival (14). A proportion of PTC patients exhibit RET/PTC rearrangement, leading to activation of RET and its downstream target MEK/ERK (15). Mutations in the genes of the Ras/Raf/MEK/ERK pathway are closely correlated with thyroid tumorigenesis and tumor invasion, particularly $\mathrm{BRAF}^{\mathrm{v} 600 \mathrm{E}}$, which is a characteristic mutation encountered in $>90 \%$ of PTC patients $(16,17)$. According to the study by Hou et al (10) the PI3K/Akt pathway plays a significant role in thyroid tumorigenesis, particularly in FTC and the progression of PTC and FTC to ATC. However, mutations of the PIK3CA gene are not common, although the activation of the PI3K/Akt pathway may be due to the amplification of the PIK3CA gene in certain thyroid cancer patients (18). The activation of the PI3K/Akt pathway may also be partially attributed to the promoter hypermethylation of phosphatase and tensin homolog (PTEN), which is a negative regulator of Akt (19).

\section{Treatment strategies for thyroid cancer}

Surgery is the first-line treatment strategy for different subtypes of thyroid cancer, whereas ${ }^{131} \mathrm{I}$ is used for the ablation of residual tumor tissue, as well as recurrent and metastatic DTCs. TSH suppression therapy is also commonly used for the long-term management of DTCs. The majority of PTCs respond well to surgery $+{ }^{131} \mathrm{I}+\mathrm{TSH}$ suppression; however, the treatment strategies for advanced thyroid cancer, including recurrence and metastasis of ${ }^{131} \mathrm{I}$-refractory PTC, ATC and MTC, are currently limited. Molecular-targeted drugs may represent a novel treatment option for advanced thyroid cancer; however, further investigation is required, which may later yield related information. Due to the increasing incidence of thyroid cancer, the management of advanced-stage disease represents an intractable issue.

\section{Role of autophagy in cancer}

Autophagy is a common process in cells, which occurs through the formation of double membrane structures that engulf cytoplasmic material and fuse with lysosomes, leading to degradation of long-lived proteins, aggregated proteins and damaged organelles, with the purpose of homeostasis maintainance and material recycling $(20,21)$. Autophagy may be triggered or enhanced by hypoxia, nutritional deprivation, radiation, chemical drugs and other stimulants. The role of autophagy in cancer remains controversial, as it appears to be involved in tumorigenesis, cancer development and treatment outcome $(22,23)$. However, due to the advances in cancer research, a consensus was gradually reached, stating that autophagy may act as a cancer suppressor or promoter, depending on the different type, grade and therapeutic strategies $(23,24)$.

Early stages of tumorigenesis. It has been confirmed in vivo that autophagy suppresses cancer development during its early stages, which may be due to the role of autophagy in maintaining genomic integrity, controlling protein quality and clearing reactive oxygen species (ROS) and other stressors (25-27). p62 is a ubiquitin- and LC3-binding protein degraded by autophagy, which is likely to accumulate when autophagy is deficient and promote tumorigenesis (27). It was demonstrated that p62 acts as a scaffold/adaptor protein in concert with tumor necrosis factor (TNF) receptor-associated factor 6 to mediate the activation of nuclear factor $\kappa$-light-chain-enhancer of activated $B$ cells in response to upstream signaling. It was confirmed that autophagy controls DNA damage, which is associated with accumulation of damaged mitochondria, endoplasmic reticulum chaperones, protein disulfide isomerases and ROS (28).

Dual role of autophagy in tumor progression. The evaluation of the role of autophagy in metastasis should take into consideration the different contextual demands placed on tumor cells during the metastatic process (23). Initially, autophagy may promote inflammation and immunity to control metastasis. However, autophagy may also avert anoikis and favor prometastasis when cancer cells detach from the extracellular matrix (23). The ability of cancer cells to invade and metastasize is closely correlated with the process of epithelial-to-mesenchymal transition (EMT) (29). It was recently demonstrated that ectopic expression of the DEDD gene in the MDA-MB-231 metastatic breast cancer cell line led to the degradation of the EMT inducers Snail and Twist through autophagy activation (30). Reversely, knockdown of DEDD in the MCF7 non-metastatic breast cancer cell line led to autophagy reduction and EMT promotion (30). 
Autophagy in cancer treatment. The role of autophagy in cancer treatment is complicated and mainly depends on the different context and treatment strategies. Breast tumor cell radiosensitization by vitamin $\mathrm{D}$ or vitamin $\mathrm{D}$ analogs is mediated through autophagy (31). However, in radioresistant MDA-MB-231 breast cancer cells, autophagy serves as a protective and pro-survival mechanism through promotion of radioresistance mediated by the autophagy inhibitors 3-methyladenine (3-MA) or chloroquine (32). Inhibition of hypoxia-induced autophagy is likely to sensitize the hypoxic breast cancer cells to ionizing radiation (33).

In conclusion, autophagy plays a dual role in cancer treatment, which may be beneficial when used as a guide for autophagy-based personalized therapeutics. Preclinical and clinical studies have used autophagy inducers (eg., NVP-BEZ235) and inhibitors (eg., hydroxychloroquine) in cancer treatment, with the aim of identifying novel therapeutic routes (22).

Mechanism of autophagy. The PI3K/Akt pathway is key to tumorigenesis and tumor cell proliferation, growth, survival and mobility (34). Abnormalities of this pathway are frequently correlated with the activation or amplification of PI3K and Akt, or PTEN loss in a variety of tumors, making them potential targets for anticancer therapy. However, another role of the PI3K/Akt pathway is autophagy suppression and activation of this pathway was shown to decrease autophagy through mTOR activation (35). The contradiction is that inhibition of this pathway is beneficial for cancer treatment; however, it potentially activates autophagy, which may be protective for cancer $(36,37)$. Thus, inhibition of the PI3K/Akt pathway by an inhibitor combined with an autophagy suppression agent has been considered for cancer treatment (38). The MAPK pathway also plays a significant role in autophagy. Due to the different role of Ras in the PI3K pathway, Ras may play a dual role in autophagy (37). When Ras activates PI3KCA, autophagy is inhibited (39); however, when it selectively activates the MAPK pathway, autophagy is stimulated (40). Several other genes may also affect the autophagy pathway, but they are not the focus of this study (22).

Correlation between autophagy and thyroid cancer. To date, several preclinical studies have demonstrated the effects of autophagy in thyroid cancer treatment. Targeting the autophagy promoter PI3KIII by 3-MA, led to autophagy suppression and decreased the PTC sensitivity to doxorubicin and external radiation (41). Targeting the autophagy negative regulator mTOR by RAD001, led to autophagy activation and enhanced the chemosensitivity and radiosensitivity of PTC (42). It appears that the activation of autophagy may be beneficial for PTC treatment, which was verified by silencing the autophagy ATG7 gene, which desensitized the PTC cells to TNF-related apoptosis-inducing ligand (TRAIL)-induced apoptosis (43). However, in ATC FRO cells, inhibition of autophagy by ATG7 silencing sensitized FRO cells to TRAIL-induced apoptosis. Thus, the results of autophagy regulation may vary in different contexts. A small molecule, termed reversine, has been reported to possess anticancer properties. Further research demonstrated that reversine suppresses the growth of human thyroid cancer and triggers autophagy through the PI3K/Akt pathway in FTC cells. With enhancement of autophagy, the anticancer effect of reversine was amplified in FTC cells (44). A kinase-targeted drug is a type of enzyme inhibitor that blocks the action of one or more protein kinases. Sorafenib and sunitinib are the classical kinase-targeted drugs, which mainly target the VEGF and MAPK pathways to inhibit angiogenesis and tumor progression. Autophagy induced by RAD001 was shown to potentiate the antiproliferative activity of sunitinib and sorafenib in MTC (45).

Although the role of autophagy in cancer has been extensively investigated, its role in thyroid cancer development and progression has not been clearly determined. In this review, we aimed to elucidate the role of autophagy in thyroid cancer and determine whether there is a correlation between the two. Hou et al (10) demonstrated that amplification of the PI3K/Akt and MAPK pathways plays a key role in the promotion of thyroid cell transformation to cancer cells and further activation of the two pathways promotes thyroid cancer aggressiveness and progression to ATC. The development of targeted drugs may provide potential strategies to inhibit these two pathways for the treatment of advanced/unresectable thyroid cancer (11). Several molecular-targeted drugs are currently in ongoing clinical trials or have already been approved for the treatment of advanced/unresectable thyroid cancer (11); however, the issue of drug resistance needs to be addressed.

Targeting the MAPK and PI3K/Akt pathways results in autophagy activation, but the potential effect is largely unknown. It was reported that inhibiting mTOR or PI3K by rapamycin and LY294002, respectively, led to enhancement of the iodine uptake ability. However, in contrast to mTOR inhibition, PI3K inhibition was able to increase iodine uptake independently of TSH $(46,47)$. These findings indicate that autophagy is closely correlated with the therapeutic sensitivity and ${ }^{131}$ I uptake, which may help with therapeutic decision making. It was recently reported that the MAPK kinase (MEK) 1 and MEK2 inhibitor selumetinib successfully reversed the refractoriness to ${ }^{131} \mathrm{I}$ in 8 of 20 patients with metastatic thyroid cancer (48).

Autophagy is potentially regulated by epigenetics in thyroid cancer. A proportion of PTC patients lose the ability to uptake iodine and develop ${ }^{131}$ I-refractory disease. This is known to occur, at least partially, due to DNA methylation or histone deacetylation. The hypermethylation of the promoter of TSH receptor and sodium iodine symporter (NIS) genes silenced the expression of these genes, which are required for iodine uptake (49). Histone deacetylase (HDAC) inhibitors may restore the function of NIS, thyroperoxidase and thyroglobulin, reverse dedifferentiation and restore ${ }^{131} \mathrm{I}$ uptake in PTC (50). Of note, HDAC inhibitors may induce caspase-independent autophagic cell death when apoptosis is inhibited $(51,52)$.

MicroRNAs (miRNAs) are short (20-22 nt) non-coding RNAs that downregulate target gene expression by binding to the untranslated region of messenger RNA. Apart from their important biofunctions, miRNAs are also valuable biomarkers for cancer diagnosis. Mounting research has identified specific miRNA profiles, mainly including miR-221/222, miR-146b, miR-21 and miR-181, in different subtypes of thyroid tumors 
(adenoma, PTC, FTC and ATC), which may be useful novel biomarkers for tumor diagnosis, progression and histological characterization (53). It was recently reported that miR-7e, miR-151-5p and miR-222 are significantly increased in the serum of PTC patients and are significantly correlated with certain clinicopathological variables (nodal status, tumor size and multifocal lesion status) (54). These dysregulated miRNAs may be of great value for targeting a number of genes that regulate fundamental cellular processes. In particular, several of these miRNAs directly or indirectly regulate the PI3K/Akt and MAPK pathways, which are central to thyroid carcinogenesis (55). The dysregulation of miRNAs in thyroid cancer may be a novel therapeutic target and the abundance of studies on miRNAs is expected to lead to a novel approach to drug development (56).

Prospectives for the role of autophagy in thyroid cancer. i) Targeting the PI $3 \mathrm{~K} / \mathrm{mTOR}$ and MAPK pathways to regulate autophagy may be beneficial through enhancing the uptake and sensitivity to ${ }^{131} \mathrm{I}$ in thyroid cancer. ii) The miRNA dysregulation in thyroid cancer, which may affect the PI3K/Akt and MAPK pathways and autophagy regulators, is a potential therapeutic target, particularly for advanced thyroid cancer, and may be used for reinduction of ${ }^{131}$ I uptake in ${ }^{131}$ I-refractory thyroid cancer. iii) The effect of autophagy on EMT may reverse the dedifferentiation phenotype and reinduce differentiation, restoring ${ }^{131} \mathrm{I}$ uptake and achieving ablation of residual tumor tissue. iv) Epigenetic mechanisms may play a dual role in reinduction of ${ }^{131} \mathrm{I}$ uptake and autophagy regulation, thus exerting a therapeutic effect. v) It should be determined whether the advantages of autophagy on molecular-targeted drugs may be used for the treatment of advanced thyroid cancer. vi) The therapeutic effect of autophagy regulators, such as chloroquine and hydroxychloroquine, should be further investigated. Autophagy inhibition using hydroxychloroquine and other autophagy regulators in combination with different therapeutic strategies has been evaluated in early-phase clinical trials on various types of tumors $(22,57)$.

\section{Conclusion}

Treatment with ${ }^{131} \mathrm{I}$ is commonly used for recurrent and metastatic thyroid cancer. However, the patients receive large doses of radiation (100-300 $\mathrm{mCi}$ ) and a proportion of patients receive ${ }^{131}$ I treatment repeatedly, with uncertain potential hazards. The enhancement of the sensitivity of thyroid cancer to ${ }^{131} \mathrm{I}$ requires further investigation, through external ionizing radiation sensitivity enhancement research and clinical application. ${ }^{131}$ I-refractory thyroid cancer and MTC also pose a therapeutic challenge. Although kinase-targeted drugs may represent a novel approach to the treatment of advanced thyroid cancer, additional treatment options are required as a substitute or supplement, since the disease eventually progresses following kinase-targeted drug treatment in the majority of patients (58). Autophagy may mediate tumorigenesis, cancer progression and treatment outcome, particularly due to its dual role in these processes. Elucidating the mechanism of autophagy is crucial for the development of anticancer strategies of maximum efficacy and minimum resistance (57).

\section{References}

1. Howlader N, Noone AM, Krapcho M, Garshell J, Neyman N, Altekruse SF, Kosary CL, Yu M, Ruhl J, Tatalovich Z, Cho H, Mariotto A, Lewis DR, Chen HS, Feuer EJ and Cronin KA (eds): SEER Cancer Statistics (Review), 1975-2010. National Cancer Institute. Bethesda, MD, http://seer.cancer.gov/csr/1975_2010/, based on November 2012 SEER data submission, posted on the SEER website April, 2013.

2. Lee J, Hwang JA and Lee EK: Recent progress of genome study for anaplastic thyroid cancer. Genomics Inform 11: 68-75, 2013.

3. Hundahl SA, Fleming ID, Fremgen AM and Menck HR: A National Cancer Data Base report on 53,856 cases of thyroid carcinoma treated in the U.S., 1985-1995 [see comments]. Cancer 83: 2638-2648, 1998.

4. Smallridge RC and Copland JA: Anaplastic thyroid carcinoma: pathogenesis and emerging therapies. Clin Oncol (R Coll Radiol) 22: 486-497, 2010.

5. Pacini F, Castagna MG, Cipri C and Schlumberger M: Medullary thyroid carcinoma. Clin Oncol (R Coll Radiol) 22: 475-485, 2010.

6. Ball DW, Jin N, Rosen DM, et al: Selective growth inhibition in BRAF mutant thyroid cancer by the mitogen-activated protein kinase kinase 1/2 inhibitor AZD6244. J Clin Endocrinol Metab 92: 4712-4718, 2007.

7. Roman S, Lin R and Sosa JA: Prognosis of medullary thyroid carcinoma: demographic, clinical, and pathologic predictors of survival in 1252 cases. Cancer 107: 2134-2142, 2006.

8. Busaidy NL and Cabanillas ME: Differentiated thyroid cancer: management of patients with radioiodine nonresponsive disease. J Thyroid Res: Feb 28, 2012 (Epub ahead of print).

9. Pfister DG and Fagin JA: Refractory thyroid cancer: a paradigm shift in treatment is not far off. J Clin Oncol 26: 4701-4704, 2008.

10. Hou P, Liu D, Shan Y, et al: Genetic alterations and their relationship in the phosphatidylinositol 3-kinase/Akt pathway in thyroid cancer. Clin Cancer Res 13: 1161-1170, 2007.

11. Liebner DA and Shah MH: Thyroid cancer: pathogenesis and targeted therapy. Ther Adv Endocrinol Metab 2: 173-195, 2011.

12. Cargnello $M$ and Roux PP: Activation and function of the MAPKs and their substrates, the MAPK-activated protein kinases. Microbiol Mol Biol Rev 75: 50-83, 2011.

13. Pacini F, Ito Y, Luster M, Pitoia F, Robinson B and Wirth L: Radioactive iodine-refractory differentiated thyroid cancer: unmet needs and future directions. Expert Rev Endocrinol Metab 7: 541-554, 2012.

14. Klein M, Picard E, Vignaud JM, et al: Vascular endothelial growth factor gene and protein: strong expression in thyroiditis and thyroid carcinoma. J Endocrinol 161: 41-49, 1999.

15. Santoro M, Chiappetta G, Cerrato A, et al: Development of thyroid papillary carcinomas secondary to tissue-specific expression of the RET/PTC1 oncogene in transgenic mice. Oncogene 12: 1821-1826, 1996.

16. Portella G, Vitagliano D, Borselli C, et al: Human N-ras, TRK-T1, and RET/PTC3 oncogenes, driven by a thyroglobulin promoter, differently affect the expression of differentiation markers and the proliferation of thyroid epithelial cells. Oncol Res 11: 421-427, 1999.

17. Salvatore G, De Falco V, Salerno P, et al: BRAF is a therapeutic target in aggressive thyroid carcinoma. Clin Cancer Res 12: $1623-1629,2006$.

18. Wu G, Mambo E, Guo Z, et al: Uncommon mutation, but common amplifications, of the PIK3CA gene in thyroid tumors. J Clin Endocrinol Metab 90: 4688-4693, 2005.

19. Alvarez-Nuñez F, Bussaglia E, Mauricio D, et al: PTEN promoter methylation in sporadic thyroid carcinomas. Thyroid 16: 17-23, 2006.

20. Xie Z and Klionsky DJ: Autophagosome formation: core machinery and adaptations. Nat Cell Biol 9: 1102-1109, 2007.

21. Rubinsztein DC, Codogno P and Levine B: Autophagy modulation as a potential therapeutic target for diverse diseases. Nat Rev Drug Discov 11: 709-730, 2012.

22. Yang ZJ, Chee CE, Huang S and Sinicrope FA: The role of autophagy in cancer: therapeutic implications. Mol Cancer Ther 10: 1533-1541, 2011.

23. Kenific CM, Thorburn A and Debnath J: Autophagy and metastasis: another double-edged sword. Curr Opin Cell Biol 22: 241-245, 2010

24. Eskelinen EL: The dual role of autophagy in cancer. Curr Opin Pharmacol 11: 294-300, 2011. 
25. White E, Karp C, Strohecker AM, Guo Y and Mathew R: Role of autophagy in suppression of inflammation and cancer. Curr Opin Cell Biol 22: 212-217, 2010.

26. Qu X, Yu J, Bhagat G, et al: Promotion of tumorigenesis by heterozygous disruption of the beclin 1 autophagy gene. J Clin Invest 112: 1809-1820, 2003.

27. Mathew R, Karp CM, Beaudoin B, et al: Autophagy suppresses tumorigenesis through elimination of p62. Cell 137: 1062-1075, 2009.

28. Duran A, Linares JF, Galvez AS, et al: The signaling adaptor p62 is an important NF-kappaB mediator in tumorigenesis. Cancer Cell 13: 343-354, 2008

29. Thiery JP, Acloque H, Huang RY and Nieto MA: Epithelial-mesenchymal transitions in development and disease. Cell 139: 871-890, 2009.

30. Lv Q, Wang W, Xue J, et al: DEDD interacts with PI3KC3 to activate autophagy and attenuate epithelial-mesenchymal transition in human breast cancer. Cancer Res 72: 3238-3250, 2012.

31. Gewirtz DA, Hilliker ML and Wilson EN: Promotion of autophagy as a mechanism for radiation sensitization of breast tumor cells. Radiother Oncol 92: 323-328, 2009.

32. Chaachouay H, Ohneseit P, Toulany M, Kehlbach R, Multhoff G and Rodemann HP: Autophagy contributes to resistance of tumor cells to ionizing radiation. Radiother Oncol 99: 287-292, 2011

33. He WS, Dai XF, Jin M, Liu CW and Rent JH: Hypoxia-induced autophagy confers resistance of breast cancer cells to ionizing radiation. Oncol Res 20: 251-258, 2012.

34. Yap TA, Garrett MD, Walton MI, Raynaud F, de Bono JS and Workman P: Targeting the PI3K-AKT-mTOR pathway: progress, pitfalls, and promises. Curr Opin Pharmacol 8: 393-412, 2008.

35. Guertin DA and Sabatini DM: Defining the role of mTOR in cancer. Cancer Cell 12: 9-22, 2007.

36. Li H, Jin X, Zhang Z, Xing Y and Kong X: Inhibition of autophagy enhances apoptosis induced by the PI3K/AKT/mTor inhibitor NVP-BEZ235 in renal cell carcinoma cells. Cell Biochem Funct 31: 427-433, 2013.

37. Kondo Y, Kanzawa T, Sawaya R and Kondo S: The role of autophagy in cancer development and response to therapy. Nat Rev Cancer 5: 726-734, 2005.

38. Fan QW, Cheng C, Hackett C, et al: Akt and autophagy cooperate to promote survival of drug-resistant glioma. Sci Signal 3: ra81, 2010.

39. Furuta S, Hidaka E, Ogata A, Yokota S and Kamata T: Ras is involved in the negative control of autophagy through the class I PI3-kinase. Oncogene 23: 3898-3904, 2004.

40. Ogier-Denis E, Pattingre S, El Benna J and Codogno P: Erk1/2-dependent phosphorylation of Galpha-interacting protein stimulates its GTPase accelerating activity and autophagy in human colon cancer cells. J Biol Chem 275: 39090-39095, 2000

41. Lin CI, Whang EE, Abramson MA, et al: Autophagy: a new target for advanced papillary thyroid cancer therapy. Surgery 146: $1208-1214,2009$.

42. Lin CI, Whang EE, Donner DB, et al: Autophagy induction with RAD001 enhances chemosensitivity and radiosensitivity through Met inhibition in papillary thyroid cancer. Mol Cancer Res 8: 1217-1226, 2010.
43. Jin SM, Jang HW, Sohn SY, et al: Role of autophagy in the resistance to tumour necrosis factor-related apoptosis-inducing ligand-induced apoptosis in papillary and anaplastic thyroid cancer cells. Endocrine 45: 256-262, 2014.

44. Lu CH, Liu YW, Hua SC, Yu HI, Chang YP and Lee YR: Autophagy induction of reversine on human follicular thyroid cancer cells. Biomed Pharmacother 66: 642-647, 2012.

45. Lin CI, Whang EE, Lorch JH and Ruan DT: Autophagic activation potentiates the antiproliferative effects of tyrosine kinase inhibitors in medullary thyroid cancer. Surgery 152: 1142-1149, 2012.

46. Kogai T, Sajid-Crockett S, Newmarch LS, Liu YY and Brent GA: Phosphoinositide-3-kinase inhibition induces sodium/iodide symporter expression in rat thyroid cells and human papillary thyroid cancer cells. J Endocrinol 199: 243-252, 2008.

47. de Souza EC, Padrón AS, Braga WM, et al: MTOR downregulates iodide uptake in thyrocytes. J Endocrinol 206: 113-120, 2010.

48. Ho AL, Grewal RK, Leboeuf R, et al: Selumetinib-enhanced radioiodine uptake in advanced thyroid cancer. N Engl J Med 368: 623-632, 2013

49. Xing M: Gene methylation in thyroid tumorigenesis. Endocrinology 148: 948-953, 2007.

50. Furuya F, Shimura H, Suzuki H, et al: Histone deacetylase inhibitors restore radioiodide uptake and retention in poorly differentiated and anaplastic thyroid cancer cells by expression of the sodium/iodide symporter thyroperoxidase and thyroglobulin. Endocrinology 145: 2865-2875, 2004.

51. Yamamoto S, Tanaka K, Sakimura R, et al: Suberoylanilide hydroxamic acid (SAHA) induces apoptosis or autophagy-associated cell death in chondrosarcoma cell lines. Anticancer Res 28: 1585-1591, 2008

52. Ellis L, Bots M, Lindemann RK, et al: The histone deacetylase inhibitors LAQ824 and LBH589 do not require death receptor signaling or a functional apoptosome to mediate tumor cell death or therapeutic efficacy. Blood 114: 380-393, 2009.

53. Marini F, Luzi E and Brandi ML: MicroRNA role in thyroid cancer development. J Thyroid Res: May 10, 2011 (Epub ahead of print). doi: 10.4061/2011/407123.

54. Yu S, Liu Y, Wang J, et al: Circulating microRNA profiles as potential biomarkers for diagnosis of papillary thyroid carcinoma. J Clin Endocrinol Metab 97: 2084-2092, 2012.

55. de la Chapelle A and Jazdzewski K: MicroRNAs in thyroid cancer. J Clin Endocrinol Metab 96: 3326-3336, 2011.

56. Gambari R, Fabbri E, Borgatti M, et al: Targeting microRNAs involved in human diseases: a novel approach for modification of gene expression and drug development. Biochem Pharmacol 82: 1416-1429, 2011.

57. Janku F, McConkey DJ, Hong DS and Kurzrock R: Autophagy as a target for anticancer therapy. Nat Rev Clin Oncol 8: 528-539, 2011.

58. Marotta V, Ramundo V, Camera L, et al: Sorafenib in advanced iodine-refractory differentiated thyroid cancer: efficacy, safety and exploratory analysis of role of serum thyroglobulin and FDG-PET. Clin Endocrinol (Oxf) 78: 760-767, 2013. 